\title{
Two Elementary Cellular Automata with a New Kind of Dynamic
}

\author{
Isabel Aguiar \\ Escola Básica Mosteiro e Cávado, Lugar da Veiguinha \\ Panoias, 4700-760 Braga, Portugal \\ Ricardo Severino \\ Department of Mathematics and Applications, University of Minho \\ Campus de Gualtar 4710-057, Braga, Portugal
}

Finite elementary cellular automata (ECAs) are studied, considering pe-
riodic and the four types of fixed boundary conditions. It is shown that
two of these automata, rules 26 and 154 , have particularly interesting
dynamics. Both these rules are in Wolfram's class 2 when subject to
periodic boundary conditions but have chaotic dynamics, typical of
Wolfram's class 3 , when we consider fixed boundary conditions $a_{\ell}=1$
and $a_{r}=0$. The same rules, when fixed null boundary conditions
$a_{\ell}=0$ and $a_{r}=0$ are used, show complex dynamics with a mixture of
order and disorder completely different from the one identified with
Wolfram's class $4:$ it grows in complexity in order to reach, in just a
few time steps, an extremely simple, almost homogeneous configura-
tion, from which the complexification starts again.

\section{Introduction}

While studying elementary cellular automaton (ECA) dynamics, Stephen Wolfram was confronted with the fact that some systems showed a long-term behavior different from any other present in continuous dynamical system theory: the observed dynamics was not a fixed point, a periodic cycle, or even a chaotic, relatively aperiodic set of configurations. The second surprise was that all these systems exhibited similar dynamics, characterized by the emergence of complicated localized structures capable of traveling along the system and of interacting with each other, immersed in a large ordered background [1]. With time, this type of behavior, named complex dynamics, revealed itself as a strong metaphor for some complicated behavior shown by artificial and natural networks of interacting elements. Again, the simulation of an extraordinary simple mathematical model triggered the identification of suggestive similarities across many different fields [2]. 
Complex dynamics are now accepted to be a mixture of both ordered and disordered characteristics, in the following sense: almost all elements of the system experience periodic dynamics, with complicated structures confined to small regions, but since these structures can travel along the system, the simple dynamics followed by a cell can abruptly be destroyed for a few instants, just to return back to the previous periodic regime. Although introduced in a different context, we could easily borrow Katherine Hayles's expression orderly disorder [3] to name the dynamics observed in these elementary cellular automata (ECAs).

The main purpose of this paper is to show that there exist cellular automata whose dynamics exhibit a mixture of ordered and disordered characteristics that are totally different from the one described above, hence proving that complex dynamics are not necessarily equivalent to orderly disorder behavior.

The rest of the paper proceeds as follows. In Section 2, we introduce some definitions and notations for finite ECAs. In Section 3, we study the dynamics of a finite ECA with certain fixed boundary conditions and conclude that it has the characteristics of chaotic Wolfram's class 3 dynamics. In Section 4, we show that with a simple change of the fixed boundary conditions, we obtain an ECA whose behavior can still be described as complex dynamics, but of a very different nature from the behavior observed for Wolfram's class 4 dynamics. In Section 5, we describe the behavior of the same rule analyzed in the two previous sections, when two other types of fixed boundary conditions are considered, and conclude that they have Wolfram's class 2 dynamics. Section 6 briefly describes the studies conducted for other rules.

\section{Finite Elementary Cellular Automata}

An ECA is an infinite linear array of sites capable of being in one of two states, which we can take to be 0 or 1 , interacting locally with its nearest neighbors. The value of each site evolves in discrete time steps, according to a fixed deterministic rule, which is the same for all sites and all time steps. This rule specifies the site's new state from its current value and the values of its closest neighbors: if we denote by $a_{i}$ the state the site $i$ assumes at a given instant, then the state in the following instant is given by

$$
a_{i}^{\prime}=\phi\left(a_{i-1}, a_{i}, a_{i+1}\right), \quad i \in \mathbb{Z},
$$

with $\phi$ a three-variable Boolean function $\phi: \mathbb{Z}_{2}^{3} \rightarrow \mathbb{Z}_{2}$. When referring to an ECA local update rule $\phi$, we use Wolfram's integer representa- 
tion [4]

$$
N_{\phi}=\sum_{k=0}^{7} \phi\left(\mathrm{n}_{k}\right) 2^{k},
$$

where $\mathrm{n}_{k}$ denotes the element of $\mathbb{Z}_{2}^{3}$ corresponding to the binary representation of $k$. Figure 1 shows a graphical representation of the elementary transition rule $N_{\phi}=26$ : on the top row are depicted all the eight possible settings for the neighborhood, and the corresponding values achieved by the central site on the next time step are given at the bottom row.

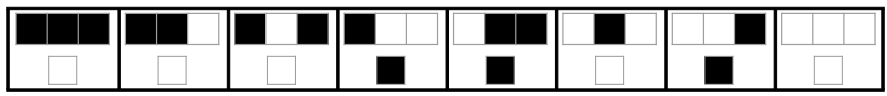

Figure 1. Graphical representation of the local update rule $N_{\phi}=26$, with the 0 and 1 states denoted by white and black squares, respectively.

In 1983, Wolfram [5] identified four basic classes of dynamics among the 256 different ECAs: the first three sort out the ECAs with long-term dynamics analogous to, respectively, a fixed point, a limit cycle, and a strange attractor, with the fourth class being reserved for the ECAs with orderly disorder dynamics. After four decades of research, this classification is believed to be valid for a much larger class of cellular automata [6].

In the following, we restrict our attention to finite ECAs, that is, to the case where we only have a finite number $n$ of sites. The finiteness of the array raises the obvious question of how to use the local update rule equation (1) for the boundary sites $a_{1}$ and $a_{n}$, that is, how to choose $a_{0}$ and $a_{n+1}$. The most popular choice, called periodic boundary conditions, simply takes $a_{0}=a_{n}$ and $a_{n+1}=a_{1}$. As can easily be seen, this choice for boundary conditions turns a finite ECA into an array of sites arranged around a circle. In this paper, we will also deal with finite ECAs with fixed boundary conditions, where $a_{0}$ and $a_{n+1}$ take fixed values $a_{0}=a_{\ell}$ and $a_{n+1}=a_{r}$, with $a_{\ell}, a_{r} \in\{0,1\}$, for all time steps.

To simplify the notation, we will place an index on the Wolfram representation of the local update rule to specify the kind of boundary conditions used: a finite ECA with local update rule $N_{\phi}$ and periodic boundary conditions will be denoted by $\left(N_{\phi}\right)_{p}$, while the representation $\left(N_{\phi}\right)_{a_{\ell}} a_{r}$ will be reserved for the case of fixed boundary conditions $a_{0}=a_{\ell}$ and $a_{n+1}=a_{r}$.

Finite cellular automata with boundary conditions other than periodic have not attracted much attention over the years. Nonetheless, in $[7,8]$, for example, results can be found on the set of attractors for 
some ECAs under fixed boundary conditions and the identification of the invertible ECAs with null boundary conditions, respectively.

When studying a finite ECA, the possibility to have a graphical representation of its dynamics yields a huge advantage. A very convenient graphical representation consists of a plot in which the configuration of the system at a particular instant is represented by a horizontal sequence of black and white squares, corresponding to 1 and 0 states, respectively, with the configurations at successive time steps shown as successive lines. This type of plot is usually referred to as a spacetime diagram of the dynamics of the cellular automaton.

In the following, we will use spacetime diagrams to study the dynamics of some finite ECAs, emphasizing the differences that occur when different types of boundary conditions are used.

As an example, Figure 2 shows a spacetime diagram of the evolution of the finite ECA rule $26_{p}$ from a randomly chosen initial configuration. A careful analysis of this plot shows that after five time steps, the system enters a cycle of period 200. The dynamics exhibited by this cellular automaton, with its short transient (the time the system needs to enter the final cycle) and the small period of its final cycle (although, in this case, proportional to the length of the system), allow us to consider the ECA rule $26_{p}$ as belonging to Wolfram's class 2 . Let us now see what happens when, instead of periodic, we choose fixed boundary conditions.

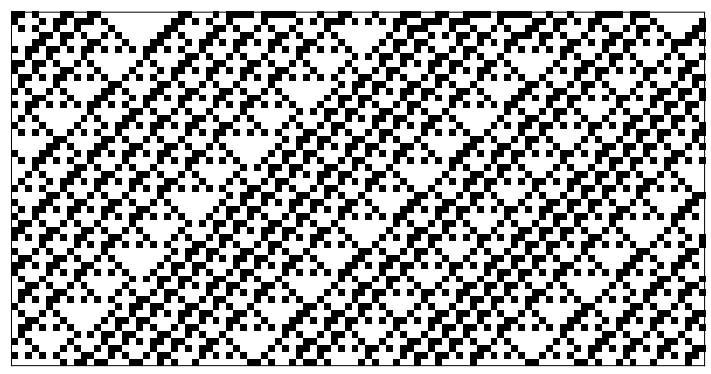

Figure 2. Spacetime diagram for the finite ECA rule $26_{p}$, from a disordered initial configuration of 100 cells, for 50 time steps.

\section{Finite Elementary Cellular Automaton Rule $\mathbf{2 6}_{\mathbf{1 0}}$}

The spacetime diagram presented in Figure 3 represents the evolution determined by the finite ECA rule $26_{10}$, from exactly the same disordered initial configuration considered before.

The spacetime diagram shows that the initial evolution of the finite ECA rule $26_{10}$ is, essentially, characterized by a left shift of patterns. However, since the system is no longer closed, there are two main dif- 
ferences relative to the dynamics of the periodic case. First, the leftshifted patterns end at the left boundary of the system; second, there are patterns generated at the right boundary of the system: triangle structures that get larger with time. These features of the initial dynamics allow us to predict that, after some time steps, the dynamics exhibited by the ECA rule $26_{10}$ will be totally different from the periodic regime observed for rule $26_{p}$. To confirm our prediction, we present in Figure 4 the continuation of the spacetime diagram for ECA rule $26_{10}$ given at Figure 3, for the next 180 time steps.

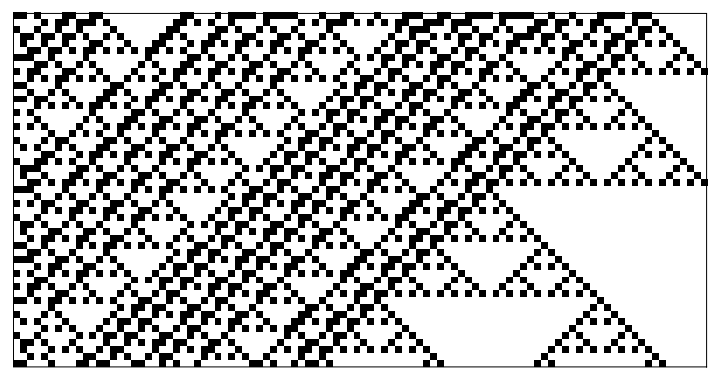

Figure 3. Finite ECA rule $26_{10}$ spacetime diagram, from the same initial configuration as in Figure 2, for 50 time steps.

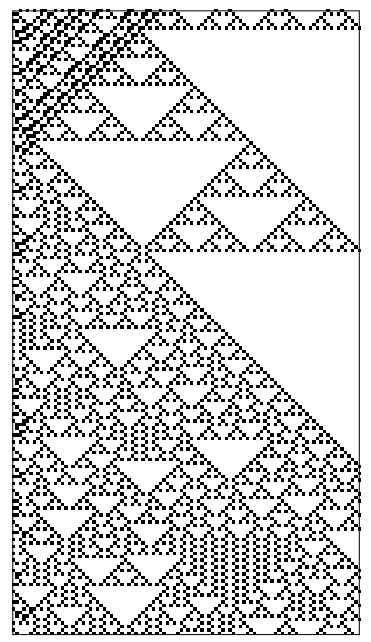

Figure 4. Continuation of the spacetime diagram for the finite ECA rule $26_{10}$ given in Figure 3, for the following 180 time steps.

The two spacetime diagrams representing the dynamics of the finite ECA rule $26_{10}$ for the first 230 time steps show that after some timeapproximately equal to two times the number of sites-the system enters a regime with complicated dynamics, characterized by triangle 
structures of different sizes generated over the entire system. In Figure 5 , we show a spacetime diagram of the dynamics of the system, 5000 time steps after the same initial configuration. This spacetime diagram suggests that the system has evolved into Wolfram's class 3 chaotic behavior. This statement can be made more precise if we study the stability of the dynamics.

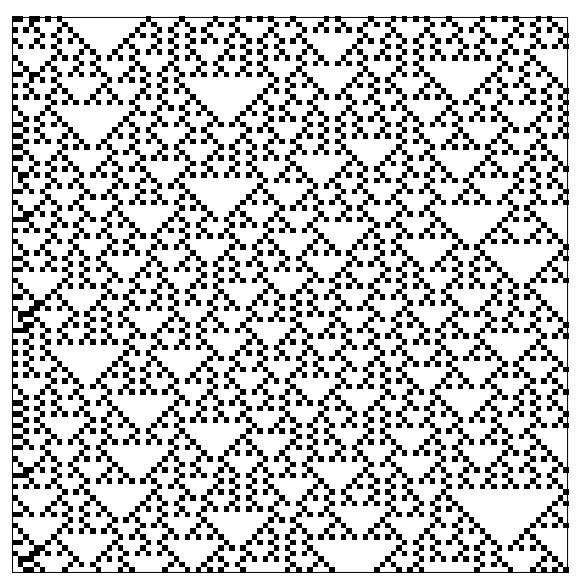

Figure 5. Finite ECA rule $26_{10}$ spacetime diagram, from the same disordered initial configuration of Figure 3, for 100 time steps, 5000 time steps after the initial time.

An important qualitative characterization of all four classes of dynamics proposed by Wolfram is provided by their sensitivity to small changes made in a configuration [9]. This is usually done by means of a difference plot, that is, a plot constructed by comparing the dynamics generated from two configurations differing in only one site and representing by black squares the sites assuming different states, all the other sites being white. Figure 6 displays the difference plot corresponding to the change in the dynamics due to a single state site modification on the first configuration shown in the spacetime diagram given in Figure 5.

The difference plot in Figure 6 confirms the chaoticity of the finite ECA rule $26_{10}$ dynamics, since the initial small perturbation spread over the entire system as time passes, a characteristic effect of ECA chaotic dynamics. Therefore, we have found a finite ECA with dynamics whose character changes dramatically when considering two different choices for its boundary conditions. 


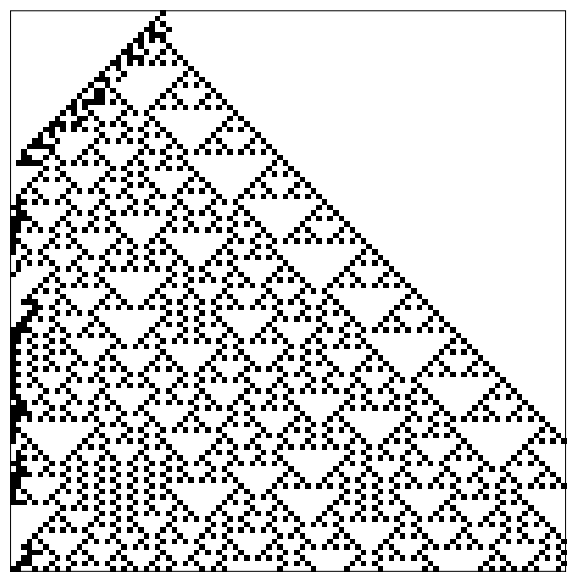

Figure 6. Difference plot corresponding to the change in the dynamics due to a single state site modification on the first configuration shown in the spacetime diagram given in Figure 5; a black square means that at that instant, the site in question is assuming different states in the two dynamics.

\section{Finite Elementary Cellular Automaton Rule $\mathbf{2 6} \mathbf{6 0}_{\mathbf{0}}$}

We now analyze the dynamics of the same local update rule $N_{\phi}=26$, but this time considering fixed null boundary conditions. In Figure 7, we show the corresponding spacetime diagram, 3900 time steps after the initial time, from the same initial configuration as considered before.

A spacetime diagram for the initial evolution of the system is very similar to the one observed for ECA rule 2610, with only a few sites near the systems' left boundaries in different states; this is something we would expect, since the systems only differ in the values for $a_{\ell}$.

The spacetime diagram in Figure 7 suggests that the dynamics evolve to a complicated triangle structure regime, typical of Wolfram's class 3 chaotic dynamics. In fact, if we compare this spacetime diagram with the one presented in Figure 5 for ECA rule 2610, it is inevitable to conclude that they look very much the same; that is, that the dynamics of $26_{00}$ seem to have entered into a chaotic regime. But let us look at the continuation of these dynamics, represented in Figure 8. A surprising change in the dynamics of the system occurs: from an evolution with chaotic characteristics, at a certain instant the system reaches an "almost homogeneous" configuration, from which starts the construction of an apparent ordered triangle structure. 


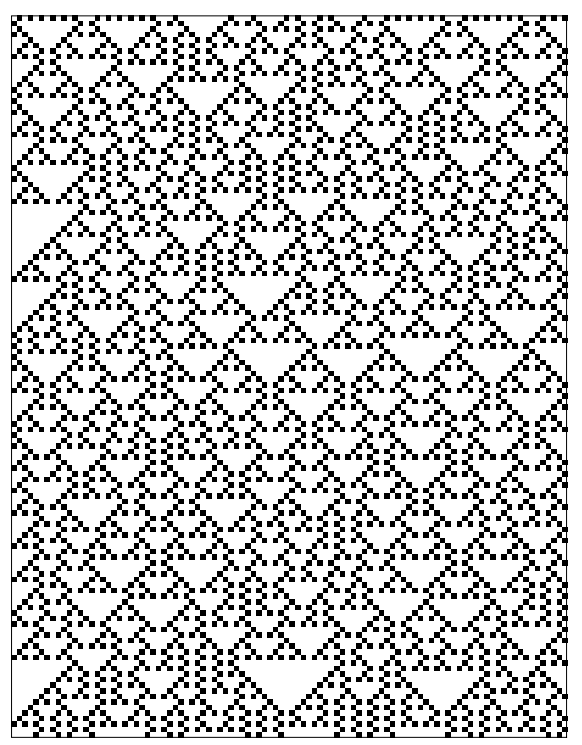

Figure 7. Spacetime diagram for the finite ECA rule $26_{00}$, for 130 time steps, 3900 time steps after the initial time, starting from the configuration considered before.

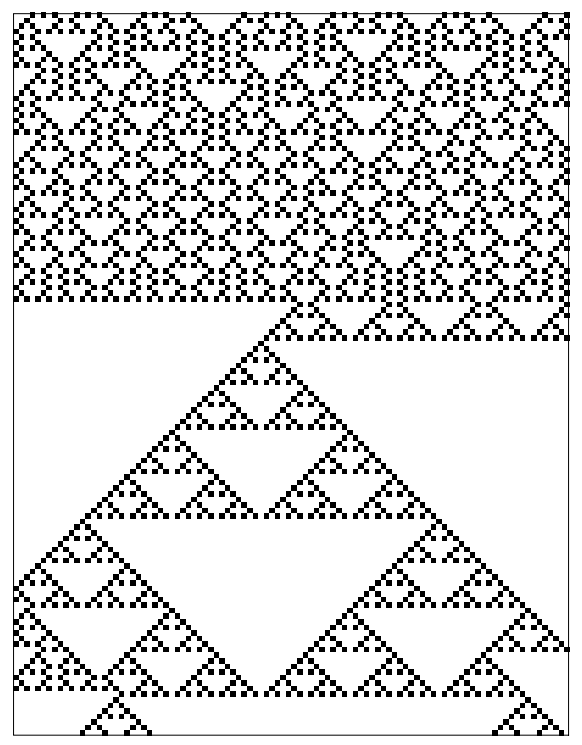

Figure 8. Spacetime diagram for the finite ECA rule $26_{00}$, for 130 time steps, immediately after the evolution represented in Figure 7. 
If we study the dynamics that follow, we observe the generation of triangles forming increasingly complicated structures and leading to a chaotic regime similar to the one shown in Figure 7, from which, once more, the systems evolve to a simplified, almost homogeneous configuration. This behavior is illustrated in Figure 9, which contains the spacetime diagram 340 time steps after the one shown in Figure 8.

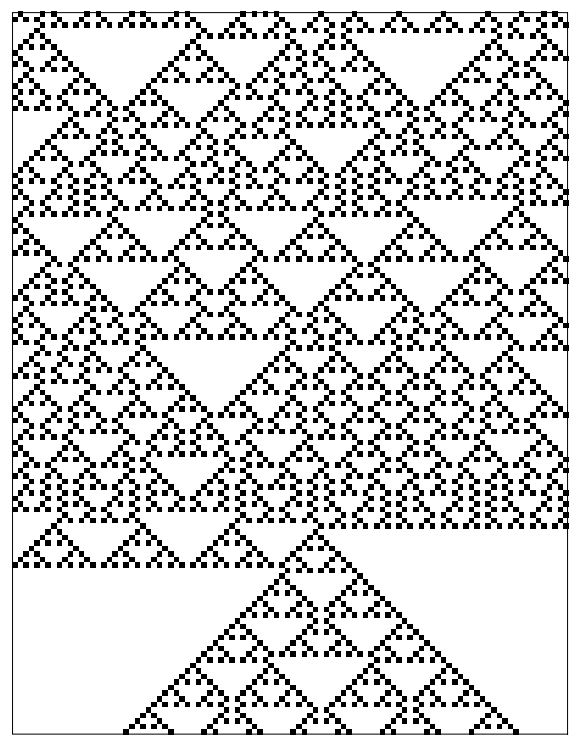

Figure 9. Spacetime diagram for the finite ECA rule $26_{00}$, for 130 time steps, 340 time steps after the evolution represented in Figure 8.

Studies conducted for other initial configurations and systems of different sizes enable us to conclude that this successive transition from disorder to order dynamics is the typical behavior of this finite ECA rule $26_{00}$.

To better illustrate this behavior, in Figure 10 we present the evolution with time of the percentage of active cells of a system with $n=3000$ sites, from a disordered initial configuration. In the same figure, for comparison purposes, we also display the same kind of plot for the finite ECA rule $26_{10}$.

A characterization of the complexity of the dynamics, as suggested in [10], can be obtained from its compressed representation. Using the Mathematica function Compress, in Figure 11 we plot the evolution with time of the length of the strings obtained from the compression of the spacetime evolution of the system, for a fixed number of time steps $\Delta t=25$. Again, the results support the idea of an increasing 
complexity of the dynamics with time and the existence of some selforganizing mechanism capable of sudden changes of the complexity of the dynamics.

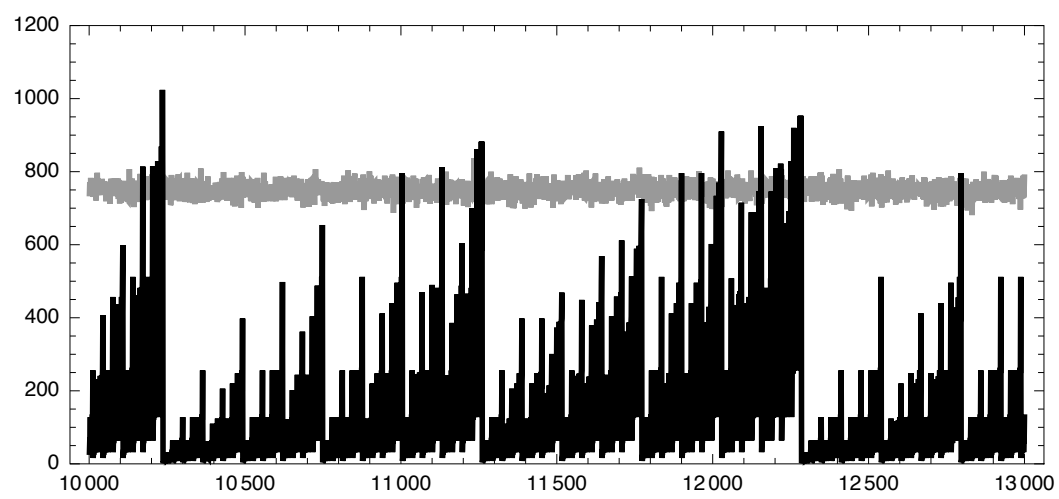

Figure 10. Evolution with time of the percentage of active cells of a system with $n=3000$ sites, from a disordered initial configuration, for ECA rules $26_{00}$ (black) and $26_{10}$ (light gray).

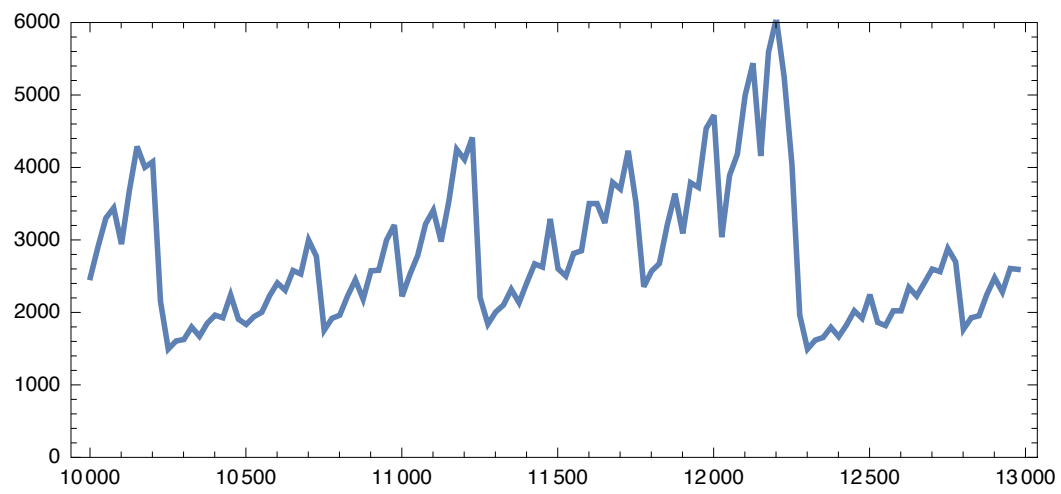

Figure 11. Evolution with time of the length of the strings obtained from the compression of the spacetime evolution of the system, for a fixed number of time steps $\triangle t=25$.

\section{Finite Elementary Cellular Automata Rules $\mathbf{2 6}_{\mathbf{0 1}}$ and $\mathbf{2 6}_{11}$}

Given the results presented in the last two sections, it is now natural to investigate the dynamics of finite ECAs with local update rule $N_{\phi}=26$ and fixed boundary conditions with state $a_{r}=1$. 
We consider first the finite ECA rule $26_{01}$. Figure 12 shows the corresponding spacetime diagram obtained from the same initial configuration as before. From this spacetime diagram, it is clear that the final dynamics of the system are, in this case, a cycle of period 5.

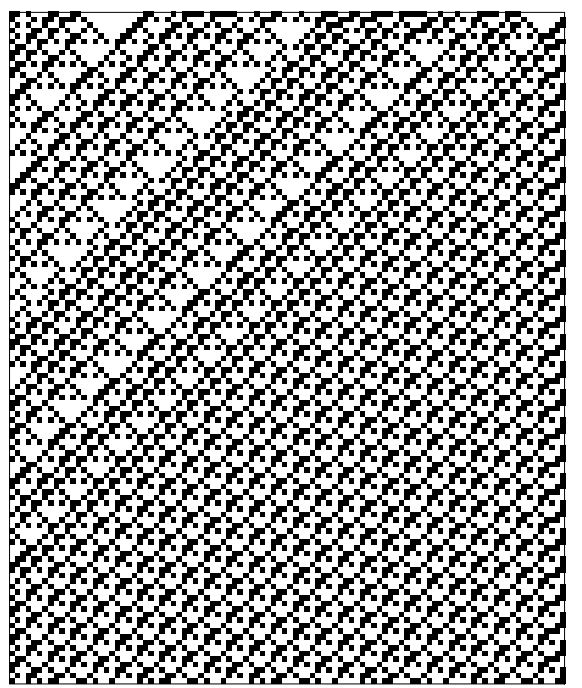

Figure 12. Spacetime diagram for the finite ECA rule $26_{01}$, for 120 time steps, from the same initial configuration as the one considered in Figure 3.

Analyzing the dynamics for a very large number of randomly chosen initial conditions, we verify that a periodic regime, with either period 3 or period 5, is the typical kind of dynamics that the system can exhibit. In other words, we conclude that the finite ECA rule $26_{01}$ belongs, as does the finite ECA rule $26_{p}$, to Wolfram's class 2 .

A similar analysis conducted for the finite ECA rule $26_{11}$ led us to conclude that this rule, starting from random initial configurations, also exhibits periodic final dynamics with small values for its period.

\section{Other Local Update Rules}

Due to the same conjugacy and reflection equivalences considered for finite ECAs with periodic boundary conditions, see [2, 11], it is a simple matter to show that in the case where we enlarge the choice of boundary conditions to include fixed boundary conditions, the number of dynamically nonequivalent rules is equal to 414 . A study analogous to the one described in the previous sections was conducted for 
all these nonequivalent rules. This study has shown that although some rules might change their dynamics when, instead of periodic boundary conditions, fixed boundary conditions are considered-for example, the rules $N_{\phi}=30,45,106$, which are in Wolfram's class 3 when periodic boundary conditions are considered, show class $2 \mathrm{dy}-$ namics for some specified fixed boundary conditions-there is only one rule with an interesting change of behavior. This is the local update rule $N_{\phi}=154$, which behaves exactly the same as rule $N_{\phi}=26$.

It is interesting to note that the outputs of the local update rules $N_{\phi}=26$ and $N_{\phi}=154$ differ at a single response: the update for the all-1 neighborhood is equal to zero for $N_{\phi}=26$ and equal to one for $N_{\phi}=154$. It is not clear, however, why even such a small difference is not meaningful in terms of the dynamics.

\section{Conclusion}

We studied all the nonequivalent finite elementary cellular automaton (ECA) rules obtained considering periodic and the four types of fixed boundary conditions. This study has shown that although some local update rules exhibit different dynamics with different choices of boundary conditions, there are two rules with a particularly interesting and unexpected change of behavior: the local update rules $N_{\phi}=26$ and $N_{\phi}=154$. Both these rules are in Wolfram's class 2 when subject to periodic boundary conditions but have a chaotic dynamics, typical of Wolfram's class 3, when we consider fixed boundary conditions $a_{\ell}=1$ and $a_{r}=0$. If we think of fixed boundary conditions as a strong restriction to the dynamics of the system, this can be seen as an unnatural result. But even more interesting is what happens when we consider fixed null boundary conditions $a_{\ell}=0$ and $a_{r}=0$. In this case, the rules show dynamics that grow in complexity in order to reach, in just a few time steps, an extremely simple, almost homogeneous configuration and start the complexification evolution again. In our view, this mixture of order and disorder is a kind of complex dynamics completely different from the one identified with Wolfram's class 4 .

This observation raises the question of knowing whether it will be necessary to impose fixed null boundary conditions on a cellular automaton in order to get the kind of complex dynamics exhibited by the rules $N_{\phi}=26$ and $N_{\phi}=154$. 


\section{References}

[1] S. Wolfram, "Computation Theory of Cellular Automata," Communications in Mathematical Physics, 96(1), 1984 pp. 15-57. doi:10.1007/BF01217347.

[2] S. Wolfram, A New Kind of Science, Champaign, IL: Wolfram Media, Inc., 2002.

[3] N. K. Hayles, Chaos Bound: Orderly Disorder in Contemporary Literature and Science, Ithaca, NY: Cornell University Press, 1990.

[4] S. Wolfram, "Universality and Complexity in Cellular Automata," Physica D, 10(1-2), 1984 pp. 1-35. doi:10.1016/0167-2789(84)90245-8.

[5] S. Wolfram, "Statistical Mechanics of Cellular Automata," Reviews of Modern Physics, 55(3), 1983 pp. 601-644.

[6] H. V. McIntosh, One Dimensional Cellular Automata, Frome, UK: Luniver Press, 2009.

[7] C. J. Twining, "The Limiting Behavior of Non-cylindrical Elementary Cellular Automata," Complex Systems, 6(5), 1992 pp. 417-432. http://www.complex-systems.com/pdf/06-5-2.pdf.

[8] S. Ghosh, N. S. Maiti, P. Pal Chaudury, and B. K. Sikdar, "On Invertible Three Neighborhood Null-Boundary Uniform Cellular Automata," Complex Systems, 20(1), 2011 pp. 417-432.

http://www.complex-systems.com/pdf/20-1-4.pdf.

[9] S. Wolfram, "Cellular Automata as Models of Complexity," Nature, 311, 1984 pp. 419-424. doi:10.1038/311419a0.

[10] H. Zenil, "Compression-Based Investigation of the Dynamical Properties of Cellular Automata and Other Systems," Complex Systems, 19(1), 2010 pp. 1-28. http://www.complex-systems.com/pdf/19-1-1.pdf.

[11] A. Wuensche and M. Lesser, The Global Dynamics of Cellular Automata, Reading, MA: Addison-Wesley, 1992. 